\title{
THE EPIDEMIC OF OBESITY IN CHILDREN AND ADOLESCENTS IN THE WORLD
}

\section{Rena I. Kosti, Demosthenes B. Panagiotakos}

Department of Food Science and Technology, Agricultural University of Athens, Athens, Greece \& Department of Nutrition and Dietetics, Harokopio University of Athens, Greece

\section{SUMMARY}

The prevalence of obesity has reached alarming levels, affecting virtually both developed and developing countries of all socio-economic groups, irrespective of age, sex or ethnicity. Concerning childhood obesity, it has been estimated that worldwide over 22 million children under the age of 5 are severely overweight, and one in 10 children are overweight. This global average reflects a wide range of prevalence levels, with the prevalence of overweight in Africa and Asia averaging well below 10\% and in the Americas and Europe above 20\%. The proportion of school-age children affected will almost double by 2010 compared with the most recently available surveys from the late 1990s up to 2003. In the European Union, the number of children who are overweight is expected to rise by 1.3 million children per year, with more than 300,000 of them becoming obese each year without urgent action to counteract the trend. By 2010 it is estimated that 26 million children in EU countries will be overweight, including 6.4 million who will be obese. Moreover, in the USA the prevalence of obesity in adolescents has increased dramatically from 5\% to 13\% in boys and from 5\% to 9\% in girls between 1966-70 and 1988-91. In this review paper we present the epidemiology of obesity in children and adolescents, including prevalence rates, trends, and risk factors associated with this phenomenon.

Key words: obesity, prevalence, risk factors, children, adolescents

Address for correspondence: Demosthenes B. Panagiotakos, 46 Paleon Polemiston St., 16674, Glyfada, Greece.

E-mail: d.b.panagiotakos@usa.net

\section{INTRODUCTION}

The prevalence of obesity has reached alarming levels, with more than 1 billion overweight adults of which 300 million are considered as clinically obese. Obesity is affecting virtually both developed and developing countries of all socioeconomic groups including all age groups thereby posing an alarming problem, described by the World Health Organization (WHO) as an "escalating global epidemic"(1). Worldwide, over 22 million children under the age of 5 are severely overweight, as are 155 million children of school age. This implies that one in 10 children worldwide are overweight (2). This global average reflects a wide range of prevalence levels, with the prevalence of overweight in Africa and Asia averaging well below $10 \%$ and in the Americas and Europe above 20\% (3). The proportion of school-age children affected will almost double by 2010 compared with the most recently available surveys from the late 1990s up to 2003 (4). In the European Union, the number of children who are overweight is expected to rise by 1.3 million children per year, with more than 300,000 of them becoming obese each year without urgent action to counteract the trend (4). By 2010 it is estimated 26 million children in EU countries will be overweight, including 6.4 million who will be obese (Table 1) (4). A potential deluge is evident across the globe with obesity rates increasing more than twofold over the past 25 years in the U.S., almost threefold in the past 10 years in England, and almost fourfold over a similar time frame in Egypt (5). Moreover, in the USA the prevalence of obesity in adolescents has increased dramatically from 5\% to $13 \%$ in boys and from 5\% to $9 \%$ in girls between 1966-70 and 1988-91 (1). In a single year from 2000 to 2001, the prevalence

Table 1. Prevalence and projections of overweight / obesity in children and adolescents in various regions of the World

\begin{tabular}{|l|l|l|l|l|}
\hline \multirow{2}{*}{ Region } & $\begin{array}{l}\text { Overweight/ } \\
\text { Obesity ** }\end{array}$ & Obesity ** & $\begin{array}{l}\text { Overweight/ } \\
\text { Obesity ** }\end{array}$ & Obesity ** \\
\cline { 2 - 5 } & & & $\begin{array}{l}\text { Projected } \\
\mathbf{2 0 1 0}\end{array}$ & $\begin{array}{l}\text { Projected } \\
\mathbf{2 0 1 0}\end{array}$ \\
\hline $\begin{array}{l}\text { Africa } \\
(1987-2003)^{*}\end{array}$ & 1.6 & 0.2 & & \\
\hline $\begin{array}{l}\text { Americas } \\
(1988-2002)\end{array}$ & 27.7 & 9.6 & 46.4 & 15.2 \\
\hline $\begin{array}{l}\text { Eastern Med } \\
(1992-2001)\end{array}$ & 23.5 & 5.9 & 41.7 & 11.5 \\
\hline $\begin{array}{l}\text { Europe } \\
(1992-2003)\end{array}$ & 25.5 & 5.4 & 38.2 & 10 \\
\hline $\begin{array}{l}\text { South East Asia } \\
(1997-2002)\end{array}$ & 10.6 & 1.5 & 22.9 & 5.3 \\
\hline $\begin{array}{l}\text { West Pacific } \\
(1993-2000)\end{array}$ & 12 & 2.3 & 27.2 & 7 \\
\hline
\end{tabular}

*There were insufficient data on school-age children in the WHO African Region to make estimates of projected prevalence rates

**In millions 
of obesity increased among U.S. adults from $19.8 \%$ in 2000 to $20.9 \%$ in 2001 (5.6\% increase) (6). If sustained at this rate over the next 10 years, the prevalence of obesity will rise by another $74 \%$, with fully one third of the US population becoming obese by 2030 (6).

This paper presents a review of the epidemiology of obesity in children and adolescents, including prevalence rates, trends, and risk factors associated with this phenomenon, in various parts of the world.

\section{DEFINITION OF CHILDHOOD OBESITY}

Obesity is a consequence of an energy imbalance - i.e., when energy intake exceeds energy expenditure over an extended period of time (7). Until recently, there has been confusion in international published work about the definition of childhood obesity, rendering comparisons of childhood obesity rates difficult (8). The most widely used measurement to define obesity is the body mass index (BMI) (weight $/$ height $^{2}, \mathrm{~kg} / \mathrm{m}^{2}$ ) where Cole et al. (9) determined values of BMI to define overweight among children, using six large nationally representative data sets drawn from population surveys.

\section{CONSEQUENCES OF OBESITY IN CHILDHOOD AND ADOLESCENCE}

As stated in the report of a WHO Consultation on Obesity (1), "overweight and obesity lead to adverse metabolic effects on blood pressure, cholesterol, triglycerides and insulin resistance. Some confusion of the consequences of obesity arises because researchers have used different BMI cut-offs, and because the presence of many medical conditions involved in the development of obesity may confuse the effects of obesity itself. The more life-threatening problems are those associated with cardio-vascular disease (CVD), conditions associated with insulin resistance, such as type 2 diabetes, certain types of cancers, and gallbladder disease. There is a range of non-fatal health problems associated with obesity, which include respiratory difficulties, chronic musculoskeletal problems, skin problems and infertility. The likelihood of developing type 2 diabetes and hypertension rises steeply with increasing body fatness. Confined to older adults for most of the 20th century, this disease now affects obese children even before puberty. Approximately $85 \%$ of people with diabetes are type 2 , and of these, $90 \%$ are obese or overweight" (1).

Overweight and obesity in childhood and adolescence are associated with a range of psychosocial and medical complications that are both immediate and long term (10) and have severe economic consequences (11).

From the psycho-social point of view, the findings of a recent study (12) showed associations of weight status with social relationships, school experiences, psychological well-being, and some future aspirations were observed. Among girls, the pattern of observations indicates that obese girls reported more adverse social, educational, and psychological correlates. Obese as well as underweight boys also reported some adverse social and educational correlates. These findings contribute to an understanding of how adolescent experiences vary by weight status and suggest social and psychological risks associated with not meeting weight and body shape ideals embedded in the larger culture. The study is a cross sectional one, of school-age adolescents (4,742 males and 5,201 females). Results showed that obese girls, when compared with their average weight counterparts, were 1.63 times less likely to associate with friends in the last week, 1.49 times more likely to report serious emotional problems in the last year, 1.79 times more likely to report hopelessness, and 1.73 times more likely to report a suicide attempt in the last year. Obese girls were also 1.51 times more likely to report being held back a grade and 2.09 times more likely to consider themselves as poor students compared with average weight girls. Compared with their average weight counterparts, obese boys were 1.91 times less likely to associate with friends in the last week, 1.34 times more likely to feel that their friends do not care about them, 1.38 times more likely to report having serious problems in the last year, 1.46 times more likely to consider themselves as poor students, and 2.18 times more likely to expect to quit school. Compared with average weight boys, underweight boys were 1.67 times more likely to report associating with friends in the last week, 1.22 times more likely to report disliking school and 1.40 times more likely to consider themselves poor students. The limitations of this study must also be considered. All measures were self-reported and consequently subject to reporting bias. Since the study is cross-sectional it is impossible and incorrect to make causal inferences of the observed associations. In addition the results are co-relational and residual confounding is always a possibility. Nevertheless, these issues are timely and may be meaningful in promoting positive social and educational experiences for all young people, as well as designing sensitive strategies for preventing and treating obesity.

On the other hand, from the medical point of view, there is an increasing amount of data showing that being overweight during childhood and adolescence is significantly associated with insulin resistance, dyslipidemia, and elevated blood pressure in young adulthood (13). Although obesity-associated morbidities occur more frequently in adults, significant consequences of obesity as well as the antecedents of adult disease occur in obese children and adolescents (14). The Bogalusa Heart Study (15) shows that childhood BMI is associated with adult adiposity, although it is possible that the magnitude of the association depends on the relative fatness of children. The most common medical consequences of obesity in youth have hyperlipidemia, glucose intolerance, hepatic steatosis and cholelithiasis while on the other hand, the less common medical consequences of obesity are hypertension, pseudotumor cerebri, sleep apnea and orthopaedic complications and polycystic ovary disease (14). In addition, findings from a study (16) conducted in Israel, showed that iron deficiency (ID) is common in overweight and obese children. The study sample included 321 children and adolescents. A significantly greater proportion of obese than normal-weight children have iron deficiency anemia (IDA) $(58,3 \%$ vs $6,7 \%)$. Insufficient dietary intake of iron, whether absolute or relative to body mass, and increased iron needs may be a result of unbalanced nutrition or repeated short-term restrictive diets. The main limitation of their study is the use of serum iron level, which was selected because it is readily available in many biochemical tests. However, infections and inflammations can decrease serum iron concentration, and there may be day-to-day variations within individuals. Because of 
potentially harmful effects of ID, obese children should be routinely screened and treated as necessary (16).

Apart from the severe health consequences of childhood and adolescence obesity, a recent study demonstrates that the obesity epidemic has deleterious economic consequences (11). Obesity is responsible for between $5 \%$ and $7 \%$ of the total annual medical expenditures in the U.S., and (11) based on an estimation of the 2001 Surgeon General report on obesity, a projection for 2003 suggested that the total costs of obesity might be as high as $\$ 139$ billion per year (11). In addition, the same study suggests that technology may be primarily responsible for the obesity epidemic. Technological advancements have allowed us to be increasingly productive at work and at home while expending fewer calories and have also reduced food prices, especially prices for energy-dense foods (11). These changes directly increase net calories and may interact with other factors (e.g., television, the built environment) to further promote weight gain (11). Obesity is not only a health but also an economic phenomenon (11). Several economic factors affect our food consumption and physical activity decisions and ultimately our weight (11).

\section{ETIOLOGY OF OBESITY}

Taking into serious consideration the severe consequences of obesity, it is of enormous importance to identify the risk factors. This task is not an easy one, since the etiology for child and adolescent obesity is not clear. Obesity is a complex condition with genetic, metabolic, behavioural and environmental factors all contributing to its development (8). However, the dramatic increase in the prevalence of obesity in the past few decades can only be due to significant changes in lifestyle influencing children and adults alike (8). These obesity-promoting environmental factors are usually referred to today under the general term of "obesogenic" or "obesigenic" (3). The current changing nature of this obesogenic environment has been well described in a WHO Technical Report (17) as follows:

"Changes in the world food economy have contributed to shifting dietary patterns, for example, increased consumption of energy-dense diets high in fat, particularly saturated fat, and low in unrefined carbohydrates. These patterns are combined with a decline in energy expenditure that is associated with a sedentary lifestyle, motorized transport, labour-saving devices at home, the phasing out of physically demanding manual tasks in the workplace, and leisure time that is preponderantly devoted to physically undemanding pastimes." Under the notion "lifestyle" are included dietary changes, changes in work and leisure patterns, cultural, behavioural, geographical, environmental, social and economic factors (17). Therefore, apart from genetic factors, the prerequisite for becoming obese is an imbalance between energy expenditure, modulated primarily by physical activity, and energy intake from foods and drinks (17).

\section{TRENDS IN DIETARY PATTERNS}

Findings from a longitudinal study (18) conducted in the U.S. showed that total fat consumption expressed as a percentage of energy intake has decreased among U.S. children.
However, this decrease is largely the result of increased total energy intake in the form of carbohydrates and not necessarily due to decreased fat consumption. The majority of children aged 5-17 years are not meeting recommendations for $\mathrm{Ca}$ intake. Much of this deficit is attributed to changing beverage consumption patterns, characterized by declining milk intakes and substantial increases in soft-drink consumption. On average, U.S. children are not eating the recommended amounts of fruits and vegetables. U.S. adolescents become less active as they get older, and one quarter of all U.S. children watch $\geq 4 \mathrm{~h}$ television each day, which is positively associated with increased BMI and skinfold thickness. Thus, the author concludes that there is an urgent need in the U.S.A. for effective prevention strategies aimed at helping children grow up with healthful eating and physical activity habits to achieve optimal growth (18).

Findings from another longitudinal study (19) conducted in Sweden in the period 1993-1999, showed that food habits change significantly during adolescence along with lifestyle changes. The sample included 208 adolescents of both gender. From the results it was derived that at 17 and 21 years of age, the adolescents consumed significantly more often pasta, vegetables, coffee and tea compared to those of age 15, while the frequency consumption of fat spread, milk, bread, potatoes, carrots and buns and biscuits decreased. The changes between age 15-17 were smaller than between age 17 and age 21 . At age 21 , the males decreased their intake of fruit, while females decreased their intake of meat. Non-meat consumers among females increased from 2 to $13 \%$. Higher educational level of the mothers of the adolescents was associated with more frequent consumption of vegetables and pasta between ages 17 and 21. Milk consumption decreased significantly in both sexes. Breakfast habits did not change: $90 \%$ had breakfast five times a week or more. Thus, it seems that adolescents during these age periods are prepared to change their dietary habits in different directions (19).

In addition, an overview (20) of current studies in the Nordic countries showed that overweight and obesity seem to become more common among adolescents, even though the prevalence figures are far from those in the U.S.A. On the other hand, dieting girls are common among adolescents, which might be a factor behind irregular meal pattern and food choices. The common smoking habits are a powerful predictor of adolescents' eating habits. The studies conducted in the Nordic countries involved adolescents aged 13 to 18 years. The results revealed that food habits are characterized by an irregular meal pattern; many adolescents skip breakfast and also the school lunch, whereas most of them have dinner. However, snacking and light meals are very common contributing $25-35 \%$ of the daily energy intake. Smoking is linked to their dietary habits as well as socio-economic conditions. Dietary intakes of vitamins and minerals are adequate for normal health and growth.

Dietary calcium intake is high whereas the intake of fibre, vitamin $\mathrm{D}$, zinc and selenium and, in girls, iron is below the Nordic recommendations. Relatively low prevalence figures of iron deficiency were found. Furthermore, there is a decrease over time in physical activity while the time spent on sedentary activities, such as television and video watching and computer games has increased during recent decades (20).

Findings from a systematic review (21) of school aged youth (10-16 years) conducted in 34 countries show that the two 
countries with the highest prevalence of overweight (pre-obese + obese) and obese youth were Malta (25.4\% and $7.9 \%)$ and the United States $(25.1 \%$ and $6.8 \%)$ while the two countries with the lowest prevalence were Lithuania $(5.1 \%$ and $0.4 \%)$ and Latvia $(5.9 \%$ and $0.5 \%)$. Overweight and obesity prevalence was particularly high in countries located in North America, Great Britain, and south-western Europe. Within most countries, physical activity levels were lower and television viewing times were higher in overweight compared to normal weight youth. In $91 \%$ of the countries examined, the frequency of sweets intake was lower in overweight than normal weight youth. Overweight status was not associated with the intake of fruits, vegetables, and soft drinks or time spent on the computer.

A total of 162,305 youth completed the survey. However, this survey has some limitations. The primary limitation of this study was that the body weights and heights were self-reported. A second limitation was that $14 \%$ of the youth surveyed did not report their height and weight. A third limitation of the study was that the dietary patterns and physical activity variables were also self-reported, and information was only obtained on the frequency and not the total volume for these variables. A final limitation of this study was that the associations observed between overweight status with dietary and physical activity patterns were based on cross-sectional data and therefore causal inferences cannot be implied. In conclusion, the study suggests that increasing physical activity participation and decreasing television viewing should be the focus of strategies aimed at preventing and treating overweight condition and obesity in youth (21).

Findings from a literature review (22) on the nutritional status and food intake in adolescents living in Western Europe showed that it is generally observed that obesity rates are increasing in young people, whereas declared energy intake is decreasing. Average daily energy input seems adequate in adolescents of Western Europe. However, fat intake, particularly saturated fat, is high while that of $\mathrm{CHO}$ and fibre is low. Proteins are mainly two-thirds from animal sources. Average micronutrient intakes correspond to recommended values in most cases, but there are a few exceptions ( $\mathrm{Ca}$ and $\mathrm{Fe}$ ) that are low, particularly in girls. Specific problems become frequent among adolescents such as dieting, smoking, getting low quality foods away from home, etc. These behaviours may induce adverse nutritional conditions. On average, nutritional problems at adolescence do not appear to be more severe than at other ages, although they may exert a strong deleterious impact on future health. Growth processes however are still continuing and nutritional inadequacies at adolescence could have a life-long health impact. Low physical activity, which is common in industrialized countries, may particularly affect the somatic and psychological development of adolescents. At this age transition in lifestyle, it is important to implement the conditions of sound nutritional and behavioural habits. The main limitation of the afore-mentioned review is that although comparisons were attempted and similarities suggested between various geographical areas, it remains difficult to develop useful, pertinent comparisons between countries, due to large methodological differences between the individual studies. Thus, the conduction of standardized, cross sectional crosscultural investigations is proposed, which could bring much valuable information and might allow relevant meaningful relationships to be established between elements of lifestyle and nutritional status in adolescence (22).

\section{TRENDS IN PHYSICAL ACTIVITY PATTERNS}

The current review examines the effect of changing physical activity patterns on the prevalence of adolescent obesity. Findings from a recent study (23) showed that decreasing sedentary behaviours can decrease energy intake in nonoverweight adolescent youth and should be considered an important component of interventions to prevent obesity and to regulate body weight. The study conducted in a sample of 16 non-overweight 12-16-year-old youth in a within-subject crossover design with three 3-week phases: baseline, increasing targeted sedentary behaviours by $25-50 \%$ (increase phase), and decreasing targeted sedentary behaviours by $25-50 \%$ (decrease phase). Specifically, targeted sedentary behaviours increased by $45.8 \%$ and decreased by $61.2 \%$ from baseline. Girls increased sedentary behaviours significantly more than did boys in the increase phase. Energy intake decreased when sedentary behaviours decreased. No significant changes in energy intake were observed when sedentary behaviours were increased. Youth also increased their activity when sedentary behaviours were decreased. One limitation of the study was the ability to include only slightly over $50 \%$ of the non-overweight adolescents because of under-reporting of energy intake. The degree of under-reporting was greater for the overweight adolescents. The degree of under-reporting was so great for overweight youth that the reported energy intakes for the overweight $(\mathrm{n}=19)$ and non-overweight $(\mathrm{n}=30)$ adolescents were approximately equal despite the fact that the overweight youth were $56.8 \%$ heavier than the non-overweight youth, which limited analysis to the non-overweight youth. Particularly because accelerometry in youth is strongly related to energy expenditure assessed by double-labelled water, an important research need for future studies is to identify methods of estimating total energy intake that are not subject to selfreporting bias, perhaps by estimating energy intake from objectively measured physical activity. A second limitation is that the phases were relatively short, and longer phases may show a different pattern of adaptation over time. Longer phases would provide the opportunity to assess the influence of changes in sedentary behaviour on body weight, which was not assessed in this study at each phase. These findings may be important for understanding how changing sedentary behaviours influence energy intake and energy balance so that more effective and targeted interventions can be developed for the prevention of youth obesity and the treatment of obese youth (23).

The association between energy intake and television viewing in adolescents was studied in a cross-sectional study (24) in which 2,546 students participated. The main finding is that on an average 1 hour of watching television equals the consumption of $653 \mathrm{~kJ}$. The adolescents in this study watched between 19 and 25 hours of television a week. Only about 3.5\% of them generally abstain from eating snacks or sweets or drinking soft drinks while they watch TV. It seems therefore safe to state that watching television is generally accompanied by the intake of food and snacks. The added energy intake is considerable. In boys it is close to $20 \%$ of their daily Average Energy Allowance (AEA), in girls it is a little less than $15 \%$. 
The question then becomes whether restricting energy intake while watching television might be a solution. If snacking and drinking energy containing drinks is a need similar to watching television, then restricting this behaviour during television viewing will only result in a shift in snacking and drinking behaviour. In such a case adolescents would find other times and other ways to indulge in these needs. This is an avenue worth investigating. If the extra energy intake is a behaviour specifically associated with watching television, then this might offer opportunities for intervention (24).

However, a Swedish study (25) refutes the speculation that reduced physical activity (PA) is associated with increased fat mass (FM). Specifically, results showed that PA was independently associated with FM in males but not in females. The data also showed an intergenerational association of FM between mothers and their daughters, but not between mothers and their sons. The study is a cross-sectional one in 445 17year-old adolescents and their mothers. According to the results, males were significantly more active than were females. PA was significantly and inversely associated with FM in males but not in females. However, FM and percentage FM in females were significantly associated with maternal FM and education level. No such associations were observed in males. Several limitations should be considered in interpreting the findings from the present study. First, it is not possible to infer a causal relation from cross-sectional data such as those in the current study. Second, the subjects may not be representative of Swedish adolescents in general. Third, self-reported PA is associated with recall bias. In conclusion, a clear sex difference was observed for the association between PA and FM in adolescents. Data also suggest a behavioural intergenerational association of FM between mothers and their daughters. Future studies, incorporating precise measures of exposures and outcome variables in parents and their offspring are needed, to test whether such an association also exists between fathers and their sons (25).

\section{OTHER RISK FACTORS ASSOCIATED WITH THE PREVALENCE OF OBESITY IN CHILDREN AND ADOLESCENTS}

This review indicates that various other risk factors are associated with the development of obesity in childhood and adolescence. The protective effect of breast-feeding against later obesity may not last through to adulthood, but obesity in later childhood is itself a predictor of adult disease, even if weight is lost and the adult is not obese (26). Therefore if breast-feeding protects against childhood obesity, that in itself may reduce the risk of adult diseases (overall morbidity and mortality from heart disease are both linked to adolescent obesity, irrespective of adult weight) (26). Furthermore, changes in maternal and, therefore, foetal nutrient supply at specific stages of gestation have the potential to substantially increase the risk of those offspring becoming obese in later life (27). The extent to which changes in dietary habits, both during pregnancy and in later life, may act to contribute to the current explosion in childhood and adult obesity still remains a scientific and public health challenge (27).

In addition, during puberty, changes in body composition occur, when girls tend to increase fat mass as a result of maturation while boys tend to increase muscle and other non- fat body mass (3).

A recent study (28) showed that parental overweight status is an important determinant of whether a child is overweight at either stage or changes from being not overweight at 5 years to becoming so at 14 years. This is a population-based prospective birth cohort study of 2,934 children who were examined at ages 5 and 14 years. The authors concluded that the results could suggest that children whose parents were overweight or obese were more likely to change from being not overweight at age 5 years to being overweight at 14 years and were more likely to be overweight at both ages. Maternal overweight status in particular was associated with these transitions. However, the study has limitations since the participation rate at both ages was $41 \%$ and the other important factors related to physical activity and diet, known to be important determinants of childhood BMI are not assessed, due to the lack of relevant information (28). The authors suggest that tackling adult obesity is likely to be important both for their own health benefit and that of their offspring and has to be taken into serious consideration in the design of intervention studies (28). In addition, apart from gender and ethnicity, the following risks factors should be mentioned (29): a) earlier adiposity rebound is associated with increased body fatness in adolescence; b) socio-economic status is another risk factor. In some developed countries, poorer children or those who live in rural settings are more at risk of obesity, whereas in countries undergoing economic transition childhood obesity is associated with a more affluent lifestyle and with living in urban regions; c) underlying medical disorders; d) prescription drugs (29).

\section{ASSOCIATIONS OF THE “MOSTLY BLAMED” DIETARY PATTERNS WITHIN THE “OBESOGENIC” ENVIRONMENT}

Let us first discuss the mostly blamed dietary patterns within the obesogenic environment. In a recent survey (30), the association between food habits and weight status was investigated in children who participated in the Bogalusa Heart Study. A 24-h dietary recall was collected over a 21year period on a cross-sectional sample of 1,562 children aged 10 year. Results show that numerous eating patterns were associated with overweight status. Particularly, consumption of sweetened beverages (58\% soft drinks, $20 \%$ fruit flavour drinks, $19 \%$ tea, and 3\% coffee), sweets (desserts, candy, and sweetened beverages), meats (mixed meats, poultry, seafood, eggs, pork, and beef) and total consumption of low-quality foods were positively associated with overweight status. The total amount of food consumed, specifically from snacks, was positively associated with overweight status. There was a lack of congruency in the types of eating patterns associated with overweight status across four ethnic-gender groups. The interaction of ethnicity and gender was significantly associated with overweight status. However, the study has the following limitations: Firstly, it was a cross sectional analysis and thus causal inferences cannot be made. Secondly, only a single 24-hour dietary recall was collected on each participant. Finally, the researchers suggest that additional studies are needed to confirm these findings in a longitudinal sample having multiple days of assessment (30). Furthermore, the findings from a prospective cohort study in the US (31) including more than 10,000 boys and 
girls aged 9-14 years, showed that the consumption of sugaradded beverages was associated with small BMI gains during the corresponding year and may contribute to weight gain among adolescents, probably due to their contribution to total energy intake. A major limitation of their study was the necessity of collecting data (including height, weight, and beverage intakes) by Food Frequency Questionnaires (FFQ) on youth by self-report on mailed questionnaires. Their FFQ did suggest portion sizes, but did not specify the number of ounces in a can or glass, so confusion over this may have further biased their estimates toward the null. In addition, authors cannot claim that the children of nurses are a representative sample of U.S. children. The study suggests that beverage intake, including limiting the consumption of soft drinks, is a potential target for diet improvement (31).

Another study (32) however refutes widespread speculation that carbohydrated soft drinks are responsible for the increase in overweight among children and adolescents. 3,111 children and adolescents of both gender participated in the study. Data from these participants from the years 1994-1996 and 1998 were collected by the U.S. Department of Agriculture. The total amount and the types of beverages consumed were analyzed according to age, race, and gender. It was found that age, race, and gender play a significant role in the total amount, types, and relative proportions of beverages consumed by children and adolescents. The relationship between body mass index (BMI) and beverage consumption is unclear. More specifically, researchers found that BMI was only related to consumption of diet carbonated beverages and milk, while those relationships were weak and that total beverage consumption and beverage choices are strongly related to age, race, and gender. Older teens tend to drink more carbonated beverages, fruit drinks/ades, and citrus juice, but less fluid milk and non-citrus juice. White adolescent boys are heavy consumers of most beverages, including carbonated soft drinks, milk, and fruit drinks/ades. BMI is positively associated with the consumption of diet carbonated beverages and negatively associated with the consumption of citrus juice. BMI was not associated with the consumption of milk, regular carbonated beverages, regular or diet fruit drinks/ades, or non-citrus juices. Finally, researchers suggest that careful monitoring of children's beverage intake is nevertheless warranted because caloric contributions must be balanced with energy expenditure (32).

Similarly the findings from a study (33) that assessed whether intake of snack foods was associated with weight change among children and adolescents refute the widespread speculation and suggest that, although snack foods may have low nutritional value, they were not an important independent determinant of weight gain among children and adolescents. In this prospective cohort study, 8,203 girls and 6,774 boys 9-14 years of age participated. The results showed that boys consumed more snack foods than girls during the entire study period. There was no relation between intake of snack foods and subsequent changes in BMI z-score among the boys, but snack foods had a weak inverse association with weight change among the girls. The association between servings per day of snack foods and subsequent changes in BMI z-score were not significant in either gender. However, the most important limitation of this study apart from the fact that weight and height information was based on self-report is that, the study does not represent a random sample of all US adolescent males and females, since the participants are children of nurses, and thus the study includes relatively few children of low socioeconomic status in the sample. Moreover, the study does not provide information on the father's weight status, thus there is incomplete information on parental weight status. Another limitation is that the study assessed snack foods, but not snacking occasions. Therefore the authors did not assess snacking on other foods, such as cereal and sandwiches, which may contribute an equal number of calories as snack foods. Since their definitions of snacking were based on types of foods eaten, not eating occasion, they may have misclassified some youth in terms of snacking patterns. It is possible that their results are therefore biased towards the null, which could explain the lack of positive association. Future studies are needed which assess snacking patterns, including snacking on items other than 'snack foods,' and the role snack foods play in overall dietary intake and weight changes. However, since most snack food items are of poor diet quality, thus regardless of the lack of association between intake of snack foods and subsequent weight gain, it would be prudent to recommend consuming snack foods only in moderation (33).

At the same time, marked changes in eating culture and behaviour have occurred at an extremely rapid pace (17). Firstly, a U.S. study showed (34) that portion sizes and energy intake for specific food types have increased markedly with greatest increases for food consumed at fast food establishments and at home. The sample of the study consists of 63,380 individuals, from two surveys, aged 2 years and older. Specifically, portion sizes vary by food source, with the largest portions consumed at fast food establishments and the smallest at other restaurants. Between 1977 and 1996, food portion sizes increased both inside and outside the home for all categories except pizza. The energy intake and portion size of salty snacks increased by $93 \mathrm{kcal}$, soft drinks by $49 \mathrm{kcal}$, hamburgers by $97 \mathrm{kcal}$, French fries by $68 \mathrm{kcal}$, and Mexican food by $133 \mathrm{kcal}$. Some potential limitations of the study are that the USDA changed its methods for collecting dietary data during the period 1989-1998 and that persons who are overweight most likely under-report their energy intake (34). Finally, the results of this study propose that control of portion size must be systematically addressed both in general as it relates to fast food pricing and marketing (34).

These observations are justified by the findings of another study (11) which mentioned that reductions in the relative price of energy-dense foods and an increased prevalence of marginal cost pricing, i.e. "supersizing" have resulted not only in an increase in food consumption between meals, but also in an increase in the amount of food consumed at each meal (11). Moreover, since it has been estimated that children are exposed to almost 10 commercials per hour of viewing, most for fast foods, soft drinks, sweets, and sugar-sweetened cereals (5), it is obvious and can be expected that television may increase demand for these products more than computer or video game use. In addition, another study (35) showed that children who increase their consumption of FFA tend to gain weight. The cohort sample consists of 7,745 girls and 6,610 boys aged 9 to 14 years, at baseline. Results showed that at baseline, frequency of eating FFA was associated with greater intakes of total energy, sugar sweetened beverages, and trans fat, as well as lower consumption of low-fat dairy foods and fruits and vegetables. Moreover, results showed that adolescents who increased their consumption of FFA over 1 year gained weight over and above the expected gain from normal growth and matu- 
ration during the adolescent period. The study also observed that cross-sectionally, adolescents who consumed greater amounts of FFA were heavier and were more likely to have poorer diet quality. However, the severe limitations of the study are the following: a) the researchers measured consumption of FFA and not fast food consumption or food purchased away from home directly; b) although they observed cross-sectional and longitudinal associations between consumption of FFA and BMI, these associations were inconsistent across age and gender; c) researchers used selfreported heights and weights to calculate BMI; d) although the participants in this study came from all 50 U.S. states, ability to generalise may be limited because the participants are sons and daughters of registered nurses and the cohort is $>90 \%$ white. The study suggests that eating large quantities of FFA, year after year, accumulates to larger weight gains that are clinically significant. Findings from this study suggest that consumption of FFA and fast foods may have pernicious effects on body weight and diet quality, and since families may eat dinners together but away from home in fast food outlets or restaurants, one public health strategy for promoting adolescent weight maintenance may be to increase nutrition education for adolescents and their parents on the importance of a well-balanced diet (35).

Another study (36) examined trends in fast-food consumption and its relationship to calorie, fat, and sodium intake in black and white adolescent girls. As it was shown, dietary intake of fast food is a determinant of diet quality in adolescent girls. In this longitudinal cohort study 2,379 black and white girls participated. Fast-food intake was positively associated with intake of energy and sodium as well as total fat and saturated fat as a percentage of calories. Fast-food intake increased with increasing age in both races. With increasing consumption of fast food, energy intake increased with an adjusted mean of 1,837 kcal for the low fast-food frequency group versus $1,966 \mathrm{kcal}$ for the highest fastfood frequency group. Total fat in the low fast-food frequency group was $34.3 \%$ as opposed to $35.8 \%$ in the highest fast-food frequency group. Saturated fat increased from $12.5 \%$ to $13 \%$ and sodium increased from $3,085 \mathrm{mg}$ to $3,236 \mathrm{mg}$ in the lowest versus the highest fast-food frequency group. These results suggest that decreasing fast-food consumption to a lower level could be a useful strategy for reducing intake of total calories and further reducing total and saturated fats. However, additional dietary strategies and changes in the food supply and market may be needed to reduce dietary sodium (36).

Finally, in developing countries and economies undergoing transition, many of the same factors may be influencing the development of obesity (8). Thus, the observed trend which combines a reduced physical activity, with significant changes in food habits and eating behaviour is of major concern.

\section{INTERVENTION STUDIES}

There are three critical aspects of adolescence that have an impact on chronic diseases, as in the case of obesity: (i) the development of risk factors during this period; (ii) the tracking of risk factors throughout life; and, in terms of prevention, (iii) the development of healthy or unhealthy habits that tend to persist throughout life (17).
This emphasis on the environmental causes of obesity leads to certain conclusions: first that the treatment for obesity is unlikely to succeed if we deal only with the child and not with the child's prevailing environment, and second that the prevention of obesity - short of genetically engineering each child to resist weight gain - will require a broad-based, public health programme (3).

A systematic review (37) suggests that following the assessment of the effectiveness of many interventions designed to prevent obesity in childhood and adolescence (individuals aged less than 18 years old) through diet, exercise, and/or lifestyle and social support are not effective in preventing weight gain, but can be effective in promoting a healthy diet and increased physical levels. The selected studies from 1990-2004 were randomised controlled trials and controlled clinical trials with minimum duration twelve weeks. The selected intervention studies employed educational, health promotion and/or psychological/family/behavioural therapy/counselling/management strategies. Twenty-two studies were included; ten long-term (at least 12 months) and twelve short-term (12 weeks to 12 months). Nineteen were school/preschool-based interventions, one was a community-based intervention targeting low-income families, and two were family based interventions targeting non-obese children of obese or overweight parents. Six of the ten long-term studies combined dietary education and physical activity interventions; five resulted in no difference in overweight status between groups and one resulted in improvements for girls receiving the intervention, but not boys. Two studies focused on physical activity alone. Of these, a multi-media approach appeared to be effective in preventing obesity. Two studies focused on nutrition education alone, but neither were effective in preventing obesity. Four of the twelve short-term studies focused on interventions to increase physical activity levels, and two of these studies resulted in minor reductions in overweight status in favour of the intervention. The other eight studies combined advice on diet and physical activity, but none had a significant impact (37).

The studies were heterogeneous in terms of study design, quality, target- population, theoretical underpinning, and outcome measures, making it impossible to combine study findings using statistical methods. In addition, there was an absence of cost-effectiveness data (37). Following the review, the absence of a sound effectiveness from the intervention studies could be justified by the fact that the length of time over which interventions are being conducted is too short to modify weight status (37). However, it is worth mentioning to include recognition of the complexity of the problem and its determinants, the sophistication of the intervention content, and the research methods required, in order to produce sound and sustainable outcome changes (37). The strongest recommendation is that all interventions are accompanied by a carefully considered evaluation design that enables sufficiently powered analysis of what is working, or not, and for whom (37). Finally, the authors recommend that a focus on short-term behaviour change is unlikely to be sustainable or effective in impacting on weight status of children and thus not an effective strategy in the absence of corresponding interventions which would impact on the sustainability of the interventions and a conducive and supporting environment (37). The reviewed interventions rarely considered the impact of parents' and family's increasingly complex working and living arrangements, yet the potential for change at the family 
level in the absence of addressing supportive strategies, is likely to be diminished (37).

Authors recommend that stakeholders (families, school environments, and others) be included in the decision making regarding the potential strategies to be implemented, and that a sustained strategy to bring about supportive environments and behaviour change in physical activity, sedentariness and healthier food choices is likely to make more of a positive impact than the interventions identified in this review (37).

\section{CONCLUDING REMARKS}

There are multiple determinants of what children eat. Among them are biological influences, parental influences and societal influences. In order to address childhood obesity, all of these factors must be considered (38). Furthermore, the available data on dietary intakes and nutritional status in populations of children and adolescents in Europe allow only limited conclusions, primarily because of a lack of consensus on methodological approaches used. Dietary studies of food and nutrient intakes across Europe using agreed and validated methodology would be of great value since there is a requirement of a consensus on concepts and approaches, definitions, age groups and other technicalities (39). Regarding the emphasis on environmental factors, the reason for corporate interest in school-age children is clear. McNeal calculated that adolescents aged 12-19 years spent $\$ 170$ billion in 2002 (40) so, it is obvious that children are heavy spenders.

It is apparent that the "obesogenic" environment appears to be largely directed at the adolescent market making healthy choices thus much more difficult (17). Food is a heavily promoted commercial product, and children are the targets. In 1997, the food industry was the second largest advertiser in the USA, with television as the most popular medium (41). Gallo notes that much television advertising is also aimed toward people who do not read newspapers, such as children.

Importantly, these advertisement dollars are spent disproportionately on highly processed and packaged foods. In 1997, nearly seven times as much money was spent advertising confectionery and snacks (i.e. candy, gum, mints, cookies, crackers, nuts, chips and other salty snacks) than was spent advertising fruits, vegetables, grains and beans (41). In the same year, the food industry spent $\$ 7$ billion on advertising; this is 21 times the $\$ 333$ million spent by the US Department of Agriculture on nutrition education the same year (41). While children are taught about nutrition in class, these few days of nutrition lessons can't possibly compete with daily exposure to advertising and vending machines outside the classroom (38). This raises the point that simply educating parents and children about the importance of good nutrition is not likely to lead to improved eating behaviour (38). Knowledge about nutrition may be less important than what foods are readily available in the environment. It may be more useful to teach parents how to implement the nutritional knowledge that they already have (38). Parents need assistance in creating an environment where primarily healthful foods are available (38). If the environment naturally provided exposure to foods that are consistent with the food guide pyramid, children would adhere to its principles more easily (38). On the other hand, a question may arise: "Could physical activity counteract an unbalanced diet?" There is a clear-cut answer: No, it is not likely. It would take more than 1-2 h of extremely vigorous activity to counteract a single large-sized (i.e. $\geq 785 \mathrm{kcal}$ ) children's meal at a fast food restaurant, and there are few children (or adults) who can maintain such a pace; moreover, the balance is only worsened if there are repeated such meals (42).

It seems that we are facing a new health problem, childhood obesity. Serious and urgent actions need to be taken from public health policy makers affecting both social and market environment in order to prevent the upcoming epidemic.

\section{REFERENCES}

1. Obesity: preventing and managing the global epidemic. Report of a WHO consultation. World Health Organ Tech Rep Ser. 2000;894:i-xii, 1-253.

2. World Heart Federation [homepage on the Internet]. Geneva: World Heart Federation [cited 2006 May 22]. Fact sheet. Children, adolescents and heart disease. Available from: http://www.worldheart.org/pdf/press. factsheets.children.pdf.

3. Lobstein T, Baur L, Uauy R; IASO International Obesity Task Force. Obesity in children and young people: a crisis in public health. Obes Rev. 2004 May;5 Suppl 1: 4-104.

4. Wang Y, Lobstein T. Worldwide trends in childhood overweight and obesity. Int J Pediatr Obes. 2006;1(1):11-25.

5. Ebbeling CB, Pawlak DB, Ludwig DS. Childhood obesity: public-health crisis, common sense cure. Lancet. 2002 Aug 10;360(9331):473-82.

6. Moore TR. Adolescent and adult obesity in women: a tidal wave just beginning. Clin Obstet Gynecol. 2004 Dec;47(4):884-9.

7. Pan American Health Organization. World Health Organization. 132nd Session of the Executive Committee; 2003 June 23-27. Washington: Pan American Health Organization; 2003.

8. Baur LA. Child and adolescent obesity in the 21st century: an Australian perspective. Asia Pac J Clin Nutr. 2002;11 Suppl 3:S524-8.

9. Cole TJ, Bellizzi MC, Flegal KM, Dietz WH. Establishing a standard definition for child overweight and obesity worldwide: international survey. BMJ. 2000 May 6;320(7244):1240-3.

10. Baur LA, O'Connor J. Special considerations in childhood and adolescent obesity. Clin Dermatol. 2004 Jul-Aug;22(4):338-44.

11. Finkelstein EA, Ruhm CJ, Kosa KM. Economic causes and consequences of obesity. Annu Rev Public Health. 2005;26:239-57.

12. Falkner NH, Neumark-Sztainer D, Story M, Jeffery RW, Beuhring T, Resnick MD. Social, educational, and psychological correlates of weight status in adolescents. Obes Res. 2001 Jan;9(1):32-42.

13. Steinberger J, Daniels SR. Obesity, insulin resistance, diabetes, and cardiovascular risk in children: an American Heart Association scientific statement from the Atherosclerosis, Hypertension, and Obesity in the Young Committee (Council on Cardiovascular Disease in the Young) and the Diabetes Committee (Council on Nutrition, Physical Activity, and Metabolism). Circulation. 2003 Mar 18;107(10):1448-53.

14. Dietz WH. Health consequences of obesity in youth: childhood predictors of adult disease. Pediatrics. 1998 Mar;101(3 Pt 2):518-25.

15. Freedman DS, Khan LK, Serdula MK, Dietz WH, Srinivasan SR, Berenson GS. The relation of childhood BMI to adult adiposity: the Bogalusa Heart Study. Pediatrics. 2005 Jan;115(1):22-7.

16. Pinhas-Hamiel O, Newfield RS, Koren I, Agmon A, Lilos P, Phillip M. Greater prevalence of iron deficiency in overweight and obese children and adolescents. Int J Obes Relat Metab Disord. 2003 Mar;27(3):4168.

17. Diet, nutrition and the prevention of chronic diseases. World Health Organ Tech Rep Ser. 2003;916:i-viii, 1-149.

18. Johnson RK. Changing eating and physical activity patterns of US children. Proc Nutr Soc. 2000 May;59(2):295-301.

19. Von Post-Skagegard M, Samuelson G, Karlstrom B, Mohsen R, Berglund L, Bratteby LE. Changes in food habits in healthy Swedish adolescents during the transition from adolescence to adulthood. Eur J Clin Nutr. 2002 Jun;56(6):532-8.

20. Samuelson G. Dietary habits and nutritional status in adolescents over Europe. An overview of current studies in the Nordic countries. Eur J Clin Nutr. 2002 Mar;54 Suppl 1:S21-8. 
21. Janssen I, Katzmarzyk PT, Boyce WF, Vereecken C, Mulvihill C, Roberts $\mathrm{C}$, et al. Comparison of overweight and obesity prevalence in school-aged youth from 34 countries and their relationships with physical activity and dietary patterns. Obes Rev. 2005 May;6(2):123-32.

22. Rolland-Cachera MF, Bellisle F, Deheeger M. Nutritional status and food intake in adolescents living in Western Europe. Eur J Clin Nutr. 2000 Mar;54 Suppl 1:S41-6.

23. Epstein LH, Roemmich JN, Paluch RA, Raynor HA. Influence of changes in sedentary behavior on energy and macronutrient intake in youth. Am J Clin Nutr. 2005 Feb;81(2):361-6.

24. Van den Bulck J, Van Mierlo J. Energy intake associated with television viewing in adolescents, a cross sectional study. Appetite. 2004 Oct;43(2):181-4.

25. Ekelund U, Neovius M, Linne Y, Brage S, Wareham NJ, Rossner S. Associations between physical activity and fat mass in adolescents: the Stockholm Weight Development Study. Am J Clin Nutr. 2005 Feb;81(2):355-60.

26. Owen CG, Martin RM, Whincup PH, Smith GD, Cook DG. Effect of infant feeding on the risk of obesity across the life course: a quantitative review of published evidence. Pediatrics. 2005 May;115(5):1367-77.

27. Budge H, Gnanalingham MG, Gardner DS, Mostyn A, Stephenson T, Symonds ME. Maternal nutritional programming of fetal adipose tissue development: long-term consequences for later obesity. Birth Defects Res C Embryo Today. 2005 Sep;75(3):193-9.

28. Mamun AA, Lawlor DA, O'Callaghan MJ, Williams GM, Najman JM Family and early life factors associated with changes in overweight status between ages 5 and 14 years: findings from the Mater University Study of Pregnancy and its outcomes. Int J Obes. 2005;29(5):475-82

29. Batch JA, Baur LA. Management and prevention of obesity and its complications in children and adolescents. Med J Aust. 2005 Feb 7;182(3):130-5.

30. Nicklas TA, Yang SJ, Baranowski T, Zakeri I, Berenson G. Eating patterns and obesity in children. The Bogalusa Heart Study. Am J Prev Med. 2003 Jul;25(1):9-16.

31. Berkey CS, Rockett HR, Field AE, Gillman MW, Colditz GA. Sugaradded beverages and adolescent weight change. Obes Res. 2004 May;12(5):778-88.
32. Forshee RA, Storey ML. Total beverage consumption and beverage choices among children and adolescents. Int J Food Sci Nutr. 2003 Jul;54(4):297-307.

33. Field AE, Austin SB, Gillman MW, Rosner B, Rockett HR, Colditz GA. Snack food intake does not predict weight change among children and adolescents. Int J Obes Relat Metab Disord. 2004 Oct;28(10):1210-6.

34. Nielsen SJ, Popkin BM. Patterns and trends in food portion sizes, 1977 1998. JAMA. 2003 Jan 22-29;289(4):450-3.

35. Taveras EM, Berkey CS, Rifas-Shiman SL, Ludwig DS, Rockett HR, Field AE, et al. Association of consumption of fried food away from home with body mass index and diet quality in older children and adolescents. Pediatrics. 2005 Oct;116(4):e518-24

36. Schmidt M, Affenito SG, Striegel-Moore R, Khoury PR, Barton B, Crawford P, et al. Fast-food intake and diet quality in black and white girls: The National Heart, Lung, and Blood Institute Growth and Health Study. Arch Pediatr Adolesc Med. 2005 Jul;159(7):626-31.

37. Summerbell CD, Waters E, Edmunds LD, Kelly S, Brown T, Campbell KJ. Interventions for preventing obesity in children. Cochrane Database Syst Rev. 2006;1:1-71.

38. Schwartz MB, Puhl R. Childhood obesity: a societal problem to solve. Obes Rev. 2003 Feb;4(1):57-71.

39. Koletzko B, de la Gueronniere V, Toschke AM, von Kries R. Nutrition in children and adolescents in Europe: what is the scientific basis? Introduction. Brit J Nutr. 2004 Oct;92 Suppl 2:S67-73.

40. Teens spent $\$ 170$ billion in 2002 [document on the Internet]. Northbrook (US): Teenage Research Unlimited; 2003 Feb 17 [cited 2005 Dec 5]. Available from: http://www.teenresearch.com/PRview.cfm?edit_id=152.

41. Gallo AE. Food advertising in the United States. In: Frazao E, editor America's Eating Habits: Changes and Consequences [monograph on the Internet]. Washington: US Department of Agriculture; 1998 [updated 1999 May 1; cited 2006 May 22]. Available from: http://www.ers.usda. gov/publications/aib750/aib750i.pdf.

42. Styne DM. Obesity in childhood: what's activity got to do with it? Am J Clin Nutr. 2005 Feb;81(2):337-8

Received May 22, 2006 Accepted June 30, 2006 